\title{
The role of dynamic contrast-enhanced MRI in differentiation of local recurrence and residual soft-tissue tumor versus post- -treatment changes
}

\author{
Lehotska V, Tothova L, Valkovic L \\ 2nd Radiologic Clinic of Medical Faculty Comenius University and St. Elisabeth Oncology Institute, Bratislava, \\ Slovakia.vlehot@ousa.sk
}

\begin{abstract}
Objectives: To evaluate the reliability of dynamic contrast-enhanced MRI in the diagnosis of local recurrence of malignant soft-tissue tumors after receiving treatment.

Methods: From March 2002 till December 2009 we performed dynamic contrast enhanced MRI in 95 patients with soft-tissue tumor after receiving treatment (surgery, radiotherapy, chemotherapy). Patients were classified according to five types of TIC.

Results: The recurrent disease was suspected in 47 patients and the biopsy was recommended. In 8 cases (TIC II), the biopsy was performed due to long-term post-treatment changes. Histological results proved STT recurrence in 45 patients; in 10 patients ( 8 with TIC II), biopsy revealed hypervascular granulation tissue, florid inflammation and reactive changes. The sensitivity for dynamic contrast-enhanced MR examination was $100 \%$, specificity $80 \%$, positive predictive value (PPV) $95.7 \%$ and negative predictive value (NPV) $100 \%$.

Conclusion: Our results indicate that TICs III, IV and V raise high suspicion of local tumor recurrence and require percutaneous imaging-guided biopsy. TIC of type II usually represents a pseudomass and the biopsy should be performed only in selected cases with increased risk of recurrent disease based on multidisciplinary approach. On the basis of literature review as well as our experiences we created a reliable algorithm proposed for diagnosing the residual or recurrent soft-tissue tumors (Tab. 2, Fig. 6, Ref. 20). Full Text in PDF www.elis.sk. Key words: soft-tissue neoplasms, residual tumor, magnetic resonance imaging.
\end{abstract}

Malignant soft-tissue tumors (STTs) macroscopically mostly appear as good demarcated masses with presence of pseudocapsule, giving an impression of benign origin. This fact may result in inadequate margins and microscopic residual nodules discovered only at histological examination, and it may be these lesions that mostly account for the frequency of later local recurrence (1). The detection of residual tumorous foci and local tumor recurrence is very difficult especially in apparent postsurgical and postirradiation changes in the surrouding tissue. Ultrasound and CT examinations are of limited value. MRI thus remains the most important method for studying and following up the residual or recurrent STTs.

The most important characteristic features of the lesion on MRI are the signal intensity (SI) and lesion configuration. As a first step for evaluating the recurrent STT, T2-weighted spin echo sequence (T2w TSE) is of highest value. T2-weighted spin echo sequence has to be associated with a fat suppression method (T2 $\mathrm{W}$ TSE FS). The recurrent soft-tissue tumor appears as a high SI lesion in T2w TSE, whereas scars usually exhibit a typically low SI

2nd Radiologic Clinic of Medical Faculty Comenius University and St. Elisabeth Oncology Institute, Bratislava, Slovakia

Address for correspondence: $\mathrm{V}$. Lehotska, $\mathrm{MD}, \mathrm{PhD}$, 2nd Radiologic Clinic of Medical Faculty Comenius University and St. Elisabeth Oncology Institute, Heydukova 10, SK-812 50 Bratislava, Slovakia.

Phone: + 421.2.59249180
(Fig. 1) because of their fibrous tissue content $(2,3,4)$. Some viable tumors containing a large amount of collagen (e.g. malignant fibrous histiocytoma, aggressive fibromatosis and fibrosarcoma) can be therefore misdiagnosed on T2w images due to their lowsignal intensity. The high SI lesions without associated mass on $\mathrm{T} 2 \mathrm{w}$ image may also cause difficulties in differential diagnosis and can be mistaken for any tissue with large amount of water content, such as hematomas of variable age, hygromas, inflammatory (Fig. 2) and irradiation changes and granulation tissue. The return period to low SI varies with extent of surgical procedure, dose of irradiation, and individual conditions influencing the reparative process. This may take months even years. Vanel et al. described tumor recurrence in a 6-month period not visible retrospectively. Either the lesion was too small or it was overlooked because it exhibited the same signal intensity as inflammatory changes and no clear mass effect (1).

The presence of high-signal intensity mass in T2w TSE always raises suspicion of tumor recurrence. In this case, intravenous administration of paramagnetic contrast agent (gadolinium chelates) is required. Absence of postcontrast enhancement can usually exclude tumor recurrence. The only exceptions are partially enhancing tumors with almost complete necrosis and minimal extent of viable tumor tissue, and tumors with large nonenhancing mucoid or myxomatous components. Soft-tissue mass with high SI on $\mathrm{T} 2 \mathrm{w}$ sequences, enhancing after intravenous injection of contrast 

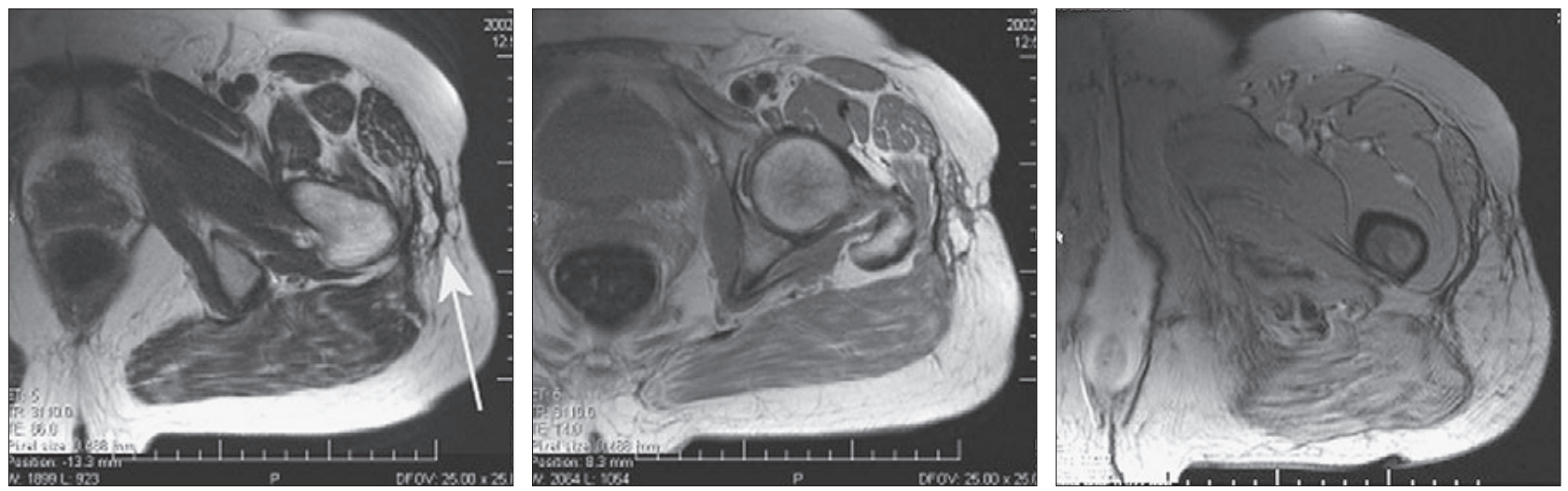

Fig. 1. Scar with fibrosis (T2w and T1w - $\downarrow$ SI irregular zone, static CE T1w - no enhancement).
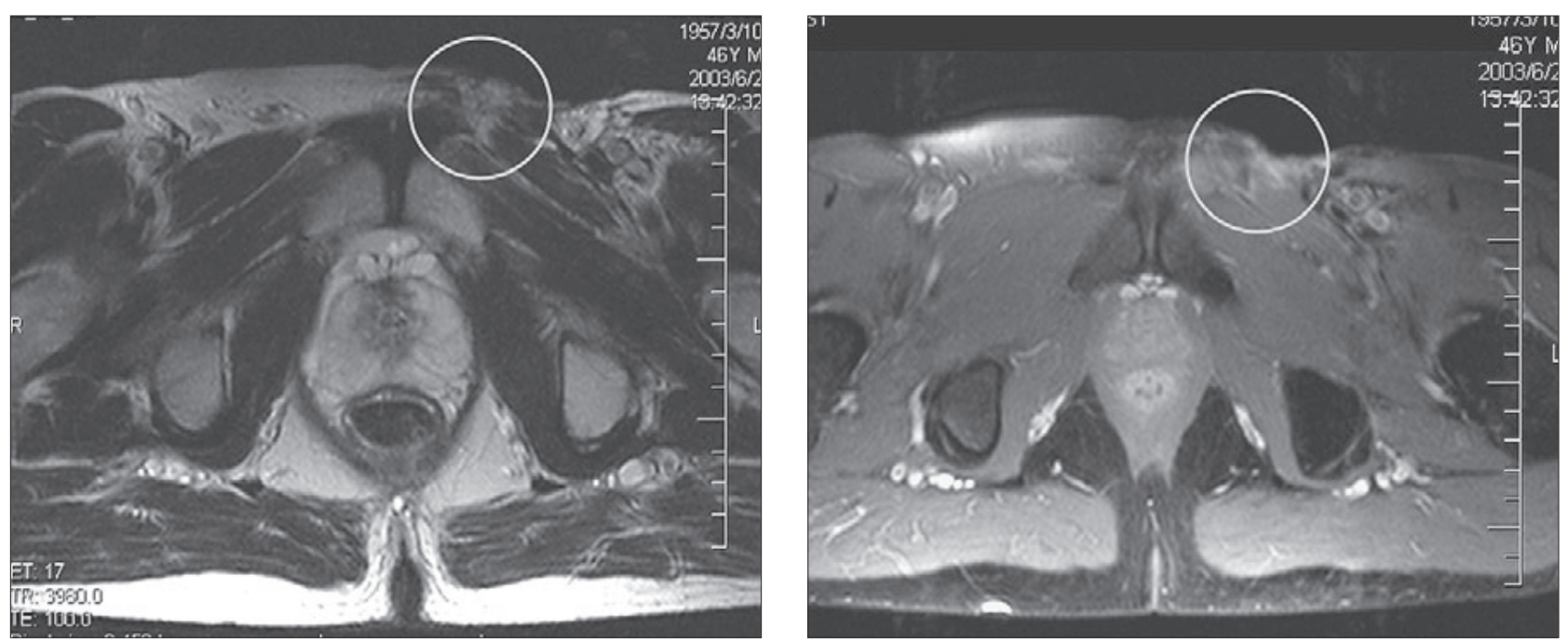

Fig. 2. Post-treatment inflammtory changes without recurrence of lipoSa.

medium, should be considered recurrent STT. However the differentiation of the inflammatory pseudotumor and variable degree of post-treatment reactive changes from recurring disease remains a problem. In these cases, the dynamic contrast-enhanced MRI is the problem-solving technique. As this process is influenced by pathologic changes in tissues, the aim of dynamic contrast-enhanced (CE) MR imaging is to detect and depict the differences in early intravascular and interstitial distribution, (5-12). One of frequently used techniques is the region of interest (ROI) technique $(13,14)$ based on dynamic analysis and graphical expression of the timedependent enhancement values with obtaining time-to-intensity curves (TIC) (Fig. 3). Active tumors show rapid enhancement in the early phase of vascularisation, whereas post-treatment changes enhance in the later period, almost after two and more minutes.

The basic principle of the dynamic CE study is to follow gadolinium pharmacokinetics after its intravenous administration. The main pieces of information obtained with this method are the characteristics of tissue vascularisation and perfusion, capillary permeability, and size and composition of interstitial space. Figure 4 shows the classification of time-to-intensity curves. The main value of this technique is in the detection of residual or recurrent STT

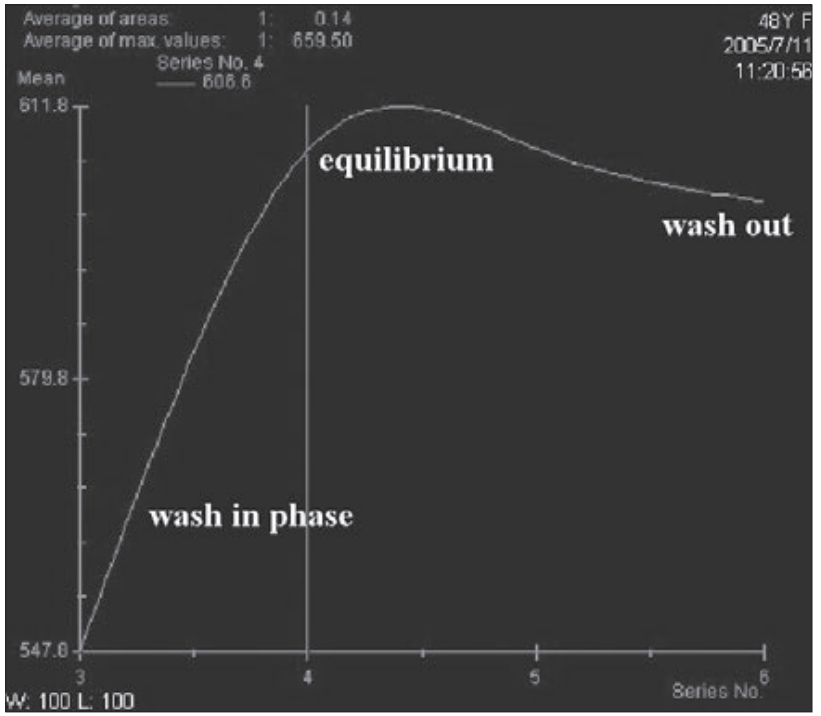

Fig. 3. Time-to-intensity curve (TIC). Temporal change of the signal intensity in a region of interest (ROI) or in a pixel is plotted against time. 

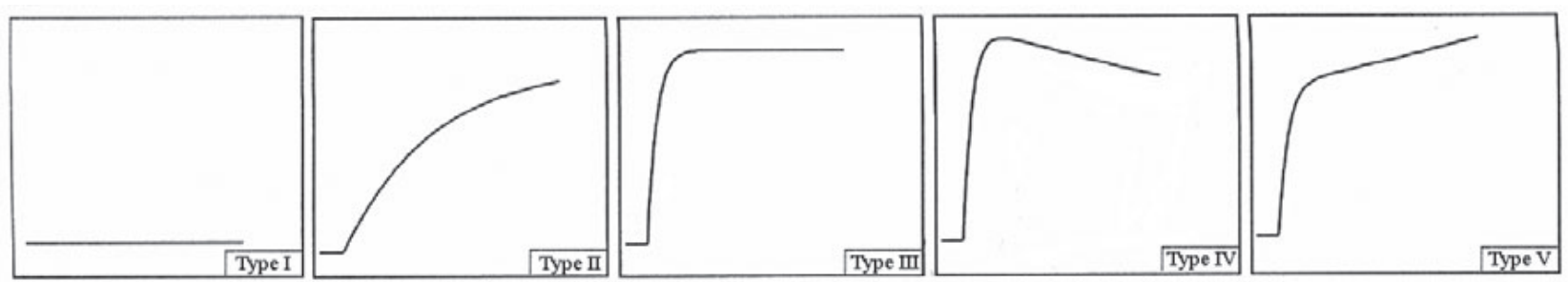

Fig. 4. Classification of time-to-intensity curves: type I - no enhancement, type II - gradual increase in enhancement, type III - rapid initial enhancement followed by plateau, type IV - rapid initial enhancement followed by a wash-out phase, type V - rapid initial enhancement followed by sustained late enhancement (30). Sensitivity $72-83 \%$, specificity $77-89 \%$ (14).

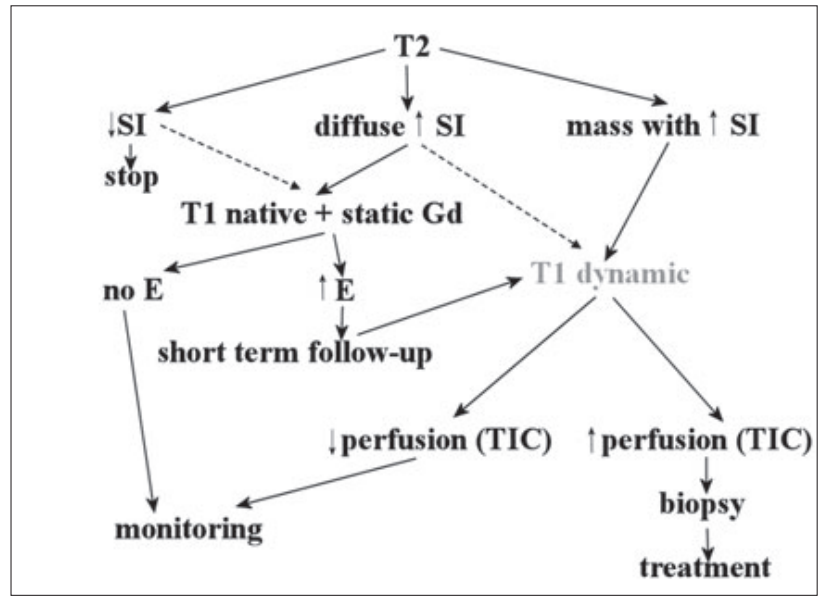

Fig. 5. Recommended diagnostic algorithm in suspicious recurrent STT.

in the region of post-treatment changes, monitoring the treatment response after neoadjuvant chemotherapy, identification of viable tumor areas before biopsy as well as in the differentiation of biological behavior of the tumorous tissue (benign versus malignant).

According to literature (15), the evaluation of malignant potential of musculoskeletal lesions with slope of TICs was possible with levels of sensitivity and specificity ranging from $72 \%$ to $83 \%$, and $77 \%$ to $89 \%$, respectively. An overlap was described among some highly vascularized or perfused benign lesions (such as capillary or high-flow hemangioma, myositis ossificans, abscess, granulation tissue, and occasionally, aggressive fibromatosis, neurinoma and neurofibroma) and malignant tumors. Combined nonenhanced static and dynamic contrast-enhanced MR imaging demonstrate the best diagnostic performance in prediction of malignancy for STT (16). After surgery, chemotherapy and radiation therapy, a residual or recurrent tumor mass may be difficult to detect as clinically as with imaging methods. This is due to scar formation and early and later post-treatment changes such as inflammation, presence of granulation tissue and development of fibrosis. Postsurgical scars tend to enhance within the fist six months. After radiation therapy, irradiated normal tissue demonstrates patchy or diffuse enhancement during the first nine months. After 10 to 18 months, the enhancement slowly decreases in a variable way.

On the basis of literature review as well as our experiences, we created a reliable algorithm proposed for diagnosing residual or recurrent soft-tissue tumors (Fig. 5). The MR imaging follow up of patients with malignant soft-tissue tumor history, after surgical treatment, radiation therapy or chemotherapy should usually begin with a T2-weighted sequence. If there is a low-signal intensity area presenting typically fibrotic changes, the examination is considered negative and could be finished with recommendation of next follow-up after 6-12 months depending on the patient's clinical status. If diffuse high-signal intensity without a mass is seen, T1weighted sequences both without and with contrast injection are required. If no contrast enhancement is present, a usual follow-up after 6-12 months is recommended. In case of static postcontrast enhancement, dynamic contrast-enhanced study in shorter time period is useful for excluding pseudomass. According to recent literature data, in these cases we always perform dynamic contrast-enhanced study followed by subtraction method. Dynamic contrast-enhanced MR imaging is the method of first choice, when on initial T2-weighted sequence a mass with high-signal intensity appears. Masses with TICs showing a slow increase in enhancement (low vascularization and low perfusion) should be observed by regular follow-ups but those with an early and fast enhancement increase (high perfusion and vascularization rates) have to undergo biopsy and treatment.

\section{Materials and methods}

From March 2002 till December 2009 we examined 95 patients with malignant soft-tissue tumors after receiving treatment (sur-

Tab. 1. Classification of examined patients according to TIC characteristics .

\begin{tabular}{lc}
\hline Dynamic contrast-enhanced study & $\begin{array}{c}\text { Patients } \\
(\mathrm{n}=95)\end{array}$ \\
\hline
\end{tabular}

No enhancement - TIC I (scar, fibrosis)

15

Gradual enhancement - TIC II

(inflammation, hyperemia, organizing hematoma)

$33^{*}$

Rapid enhancement with plateau - TIC III

(suspicious recurrence - biopsy required)

Rapid enhancement with wash-out - TIC IV (high suspicion - biopsy)

Rapid enhancement with sustained later increase - TIC V (high suspicion - biopsy)

16

* In 8 cases, biopsy was recommended because of long-term persistent changes $>6$ months after surgery and/or $>12$ months after radiotherapy) 


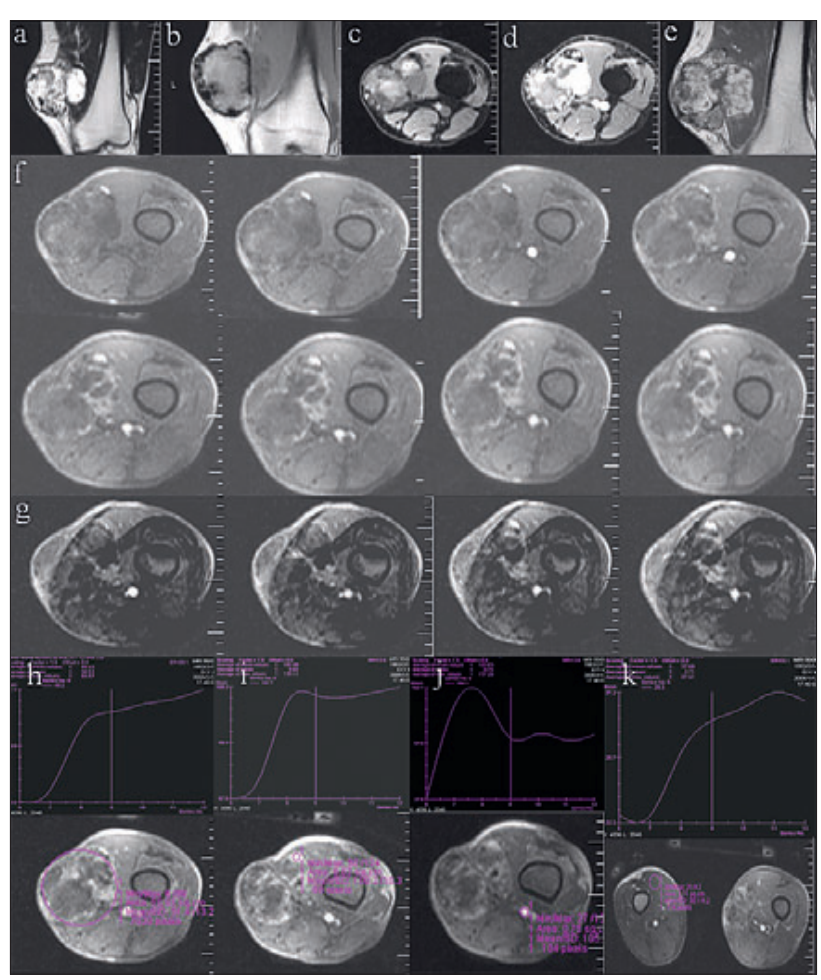

Fig. 6. A 54-year old man with malignant fibrous histiocytoma in the left knee region.

Static sagittal turbo spin-echo T2-weighted image a) shows a lobulated mass with predominantly high-signal intensity areas. On sagittal spin-echo T1-weighted image b) the tumor is of homogeneously low signal intensity. On axial fat suppressed image c) the inhomogeneous tumor mass is well seen. On axial contrast-enhanced fat suppressed static. T1-weighted image d) and on T1-weighted spin-echo image without fat suppression e) the tumor enhancement is inhomogeneous, differentiating the viable tumor components and necrosis. Dynamic contrast-enhanced study f) and subtraction images g) depict the most enhancing parts of the tumor. Time-to-intensity curves of whole tumor h), a fast enhancing area of the tumor i), of popliteal artery j) and of the healthy muscle $k$ ) are shown.

gery, radiotherapy or chemotherapy). There were 54 males (median age 51 years) and 41 females (median age 53 years). Patients were classified according to five types of TIC (Tab. 1). The technique of dynamic contrast-enhanced MR examination requires a bolus of gadolinium contrast medium in amount of $0,1-0,2 \mathrm{ml} / \mathrm{kg}$ followed immediately by saline flush of about $20 \mathrm{ml}$ at an injection rate of $3-5-\mathrm{ml} / \mathrm{sec}$. Use of a power injector is preferable. T1-weighted 3D turbo flash and/or fat suppressed T1-weighted 3D turbo flash (Siemens, Erlangen, Germany) sequences followed with subtraction imaging are usually performed. Although fat saturation might be useful, in practice this is not necessarily done, as fat is adequately suppressed by most postprocessing techniques, e.g. in subtraction and first-pass images $(11,12,17,18)$. The delay between the native sequence and first-pass dynamic postcontrast sequence is about 7-15 seconds (11). The range of temporal resolution is one image per 3.5-7 seconds till 90-180 (240) seconds after contrast administration, matrix size $128 \times 128$, the number of measurements of 5-10 is thus mandatory. An important role belongs to the
Tab. 2. Test of reliability for dynamic CE study in diagnosis of STT recurrence.

\begin{tabular}{lcc}
\hline & Biopsy-proven malignancy & Biopsy-proven benignity \\
\hline MRI-positive & 45 & $2(\mathrm{FP})$ \\
MRI-negative & $0(\mathrm{FN})$ & 8 \\
\hline
\end{tabular}

qualitative "subtraction method", in which the precontrast image is subsequently subtracted from all postcontrast image series of the dynamic study. In this way, discrete, early enhancing areas are detected, and high signals from fat and hemorrhage are nullified (15). It helps to evaluate the time interval between the onset of arterial and tumoral enhancement and to detect the viable part of the lesion. This is of high importance for indicating the best site for biopsy or to determine the degree of response to preoperative chemotherapy $(6,19,20)$.

\section{Results}

Biopsy was performed altogether in 55 of 95 examined patients with malignant soft-tissue tumor after receiving treatment (TIC III, IV, V and 8 with TIC II). Due to TIC classification, recurrent disease was suspected in 47 patients and the biopsy was required. In 8 cases (TIC II), the biopsy was performed due to long-term post-treatment changes. Histology proved STT recurrence in 45 patients; in 2 patients, hypervascular granulation tissue was found. The 8 TIC II classified patients turned out to be florid inflammatory and reactive changes in histological results. Of the 45 STT recurrent patients, 27 were males and 18 females. Histological outcomes proved 19 malignant fibrous histiocytomas (Fig. 6), 9 liposarcomas, 8 synovial sarcomas, 3 chondrosarcomas (soft tissue), 3 leiomyosarcomas, 2 fibrosarcomas and 1 lymfangiosarcoma. The most common locality was in extremities $36(80 \%)$, then retroperitoneum 7 (15.6\%), and 2 STTs were localized (4.4\%) in viscera. This means sensitivity for dynamic contrast-enhanced MR examination of $100 \%$, specificity of $80 \%$, positive predictive value (PPV) of $95.7 \%$, and negative predictive value (NPV) of 100 $\%$. Test of reliability for dynamic CE study in diagnosis of STT recurrence compared with histological results is shown in Table 2.

\section{Discussion}

Dynamic contrast-enhanced MR imaging followed by image subtraction and qualitative analysis by TICs:

1. provides clinically useful information about vascularisation and perfusion of tissue, its capillary permeability, and size and composition of interstitial space,

2. allows narrowing down the spectrum of differential diagnosis, 3. contributes to the prediction of biological behavior of the lesion, 4. is helpful for identifying viable areas of the tumor, the fact of which is of highest importance for planning the biopsy procedures,

5. is useful in achieving qualitative assessment of tumor response during and after neoadjuvant or adjuvant chemotherapy,

6. depicts residual or recurrent soft-tissue tumor foci with relatively high sensitivity and allows their differentiation against post- 
treatment changes such as fibrosis, inflammatory pseudomass, hematomas and hygromas.

Our results indicate that TICs III, IV and V highly raise suspicion of local tumor recurrence and require percutaneous imaginingguided biopsy. TIC type II usually represents pseudomass and the biopsy should be performed only in selected cases with increased risk of recurrent disease based on multidisciplinary approach.

\section{References}

1. Vanel D, Shapeero LG, Guinebretiére JM, Lecesne A, Genin J. MRI in the folloe-up of malignant and agressive soft-tissue tumors. 455-462. In: De Shepper AM, Parizel PM, De Beuckeleer L, Vanhoenacker F. Imaging of soft-tissue tumors. Springer-Verlag Berlin Heidelberg, 2001.

2. Ebner F, Kressel HY, Mintz MC, Carlson JA, Cohen EK, Schiebler M, Gefter WB, Axel I. Tumor recurrence versus fibrosis in the female pelvis: differentiation with MR imaging at 1,5 T, 1988; 166: 333 .

3. Glazer HS, Lee JK, Levitt RJ. Radiation fibrosis differentiation from recurrent tumor by MR imaging. Radiology, 1985; 756: 721-726.

4. Vanel D, Shapeero LG, De Baere T, Gilles R, Tardivon A, Genin J. Musculoskeletal tumors: follow-up with MR imaging after treatment with surgery and radiation therapy, Radiology, 1987; 164:2 43-245.

5. Dean P, Kormano M. intravenous bolus of 1251 labeled meglumine diatrizoate: early extravascular distribution. Acta radiol diagn 1977; 18 : 293-304.

6. De Baere T, Vanel D, Shapeero L, Charpentier A, Terier P, di Paola M. osteosarcoma after chemotherapy: evaluation with contrast material enhanced subtraction MR imaging, Radiology 1992; 185: 587-592.

7. Kormano M, Dean PB. Extravascular contrast material: the major component of contrast enhancement. Radiology 1976; 121: 379-382.

8. König H, Sieper J, Wolf K. Rheumatoid arthritis: evaluation of hypervascular and fibrous pannus with dynamic MR imaging enhanced with Gd - DTPA. Radiology 1990; 176: 473-477.

9. König H, Sieper J, Wolf K. Dynamic MRI for the differentiation of inflammatory joint lesions. Fortschr Roentgenstr 1990; 153: 1-5.

10. Ross J, Delamarter R, Hueftle M, Masaryk TJ, Aikawa M, Darter J, van Dicke C. Gadolinium-DTPA - enhanced MR imaging of the postoperative lumbar spine: time course and mechanism of enhancement. AJNR Am J Neuroradiol 1989; 10: 37-46.
11. Verstraete KL, Dierick A, De Deene Y, Uyttendaele D, Vandamme F, Roels H, Kunnen M. First- pass images of musculoskeletal lesions: a new and useful diagnostic application of dynamic contrast-enhanced MR imaging. Magn Reson Imaging 1994; 12: 687-702.

12. Verstraete KL, De Deene Y, Roels H, Dierick A, Uyttendaele D, Kunnen M. Benign and malignant musculoskeletal lesions: dynamic contrast-enhanced MR imaging - parametric "first-pass" images depict tissue vascularisation and perfusion. Radiology 1994; 192: 835-843.

13. Rubin DA, Kneeland JB. MR imaging of the musculoskeletal system: rechnical considerations for enhancing image quality and diagnostic yield. AJR Am J Roentgenol 1994; 163: 1155-1163.

14. Totty WG, Murphy WA, Lee JK. Soft - tissue tumors MR imaging. Radiology 1986; 160: 135-141.

15. Verstraete KL, van der Woude HJ. Dynamic contrast-enhanced magnetic resonance imaging. 83-104. In: De Shepper AM, Parizel PM, De Beuckeleer L, Vanhoenacker F. Imaging of soft-tissue tumors. Springer Verlag Berlin Heidelberg, 2001.

16. Rijswijk CSP, Geirnaerdt MJA, Taminiau AHM, Coevorden F, Zwinderman AH, Pope TL, Bloem JL. Soft-tissue tumors: value of static and dynamic gadopentetate dimeglumin-enhanced MR imaging in prediction of malignancy. Radiology 2004; 233: 493-502.

17. Van der Woude HJ, Bloem JL, Verstraete KL, Taminiau A, Nooy M, Hogendoorn P. Osteosarcoma and Ewing's sarcoma after neoadjuvant chemotherapy: value of dynamic MR imaging in detecting viable tumor before surgery. AJR Am J Roentgenol 1995; 165: 593-598.

18. Verstraete KL, Vansieleghem B, De Deene Y, Palmans H, De Greef D, Kristoffersen DT, Uytendaele D, Roels H, Hamers J, Kunnen M. Static, dynamic and first-pass imaging of musculoskeletal lesions by use of gadodiamide injection. Acta Radiol 1995; 36. 27-36.

19. Vanel D, Shapeero L, De Baere T, Gilles R, Tardivon A, Genin J, Guinebretiäre JM. MR imaging in the follow-up of malignant and aggressive soft-tissue tumors: results of 511 examinations. Radiology 1994; 190: $263-268$.

20. Van der Woude H, Verstraete KL, Taminiau A, Hogendoorn P, Vansieleghem B, Bloem J. Double slice dynamic contrast-enhanced subtraction MR images in 60 patients with musculoskeletal tumors or tumorlike lesions (abstract). Eur Radiol 1995; 5: Suppl: 181.

Received April 5, 2011. Accepted August 18, 2012. 